\title{
Probing the 14-3-3 isoform-specificity profile of interactions stabilized by fusicoccin A
}

\author{
Ananya Sengupta, Josue Liriano, Ewa A. Bienkiewicz, Brian G. Miller and James H. Frederich*
}

\section{Supporting Information Placeholder}

\begin{abstract}
Fusicoccin A (FC) is a fungal phytotoxin that stabilizes protein-protein interactions (PPIs) between 14-3-3 adapter proteins and their phosphoprotein interaction partners. In recent years, FC has emerged as an important chemical probe of human 14-3-3 PPIs implicated in cancer and neurological diseases. These previous studies have established the structural requirements for FC-induced stabilization of 14-3-3•client phosphoprotein complexes; however, the effect of different 14-3-3 isoforms on FC activity has not been systematically explored. This is a relevant question for the continued development of FC variants because there are seven distinct isoforms of 14-3-3 in humans. Despite their remarkable sequence and structural similarities, a growing body of experimental evidence supports both tissue-specific expression of 14-3-3 isoforms and isoform-specific functions in vivo. Herein, we report the isoform-specificity profile of FC in vitro using recombinant human 14-3-3 isoforms and a focused library of fluorescein-labeled hexaphosphopeptides mimicking the C-terminal 14-3-3 recognition domains of client phosphoproteins targeted by FC in cell culture. Our results reveal modest isoform preferences for individual client phospholigands and demonstrate that FC

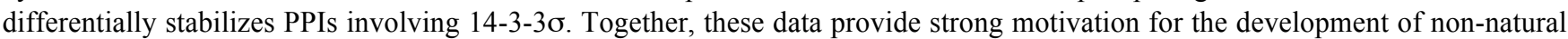
FC variants with enhanced selectivity for individual 14-3-3 isoforms.
\end{abstract}

Proteins are the main executers of cellular biochemistry, and while some proteins carry out their roles independently, most require contacts with other proteins for proper function. The resultant protein-protein interactions (PPIs) are often integrated with posttranslational modifications to form multifaceted signaling networks. ${ }^{1,2}$ The 14-3-3-protein family exemplifies this type of dynamic system. 14-3-3 proteins are phospho-binding molecules that form PPIs with a group of at least 500 client proteins that includes enzymes, receptors and transcription factors. ${ }^{3}$ This diverse interactome is directed by phosphorylation of the client at serine or threonine residues, typically within the consensus sequence RXXpZXP (X = any residue, $\mathrm{pZ}=$ phosphorylated $\mathrm{S}$ or $\mathrm{T}$ ), although alternative recognition motifs exist. ${ }^{4,5}$ Binding of the client phosphoprotein occurs along an extended amphipathic groove on the surface of 14-3-3. The effect of 14-3-3 binding is variable depending on the nature of the client and serves to finalize phosphorylation-based, signal-induced changes. In this regard, interaction with 14-3-3 can modify cellular compartmentalization, protein trafficking, or block interaction sites for other effector proteins. ${ }^{6}$ In other settings, 14-3-3 proteins constrain binding partners into an active/inactive conformation or serve as scaffolding to connect two client phosphoproteins together. ${ }^{7,8}$ The biochemistry of $14-3-3$ is intrinsically complex; however, dysregulation of 14-3-3 function is recognized as a contributor to the pathobiology of cancer and certain neurological diseases. 9,10 As such, the design of chemical tools to resolve the roles of individual 143-3 PPIs in modifying disease pathways has become an important objective. ${ }^{11}$

The natural product fusicoccin A (FC) provides one attractive entry point to 14-3-3 PPI modulators (Figure 1). ${ }^{12}$ This diterpene glycoside is a phytotoxin produced by the fungus Fusicoccum amygdali that damages infected plants via stabilization of PPIs formed between plant 14-3-3 isoforms and phosphorylated plasma membrane $\mathrm{H}^{+}$ATPase. ${ }^{13,14}$ Binding of FC enhances the lifetime of this regulatory protein complex by $\sim 90$-fold, leading to significant alterations in stoma dynamics. Intriguingly, FC also possesses pro-apoptotic and neuroprotective activity in cell culture. ${ }^{15,16}$ Human 14-3-3 PPIs directly contribute to this pharmacology, as evidenced by

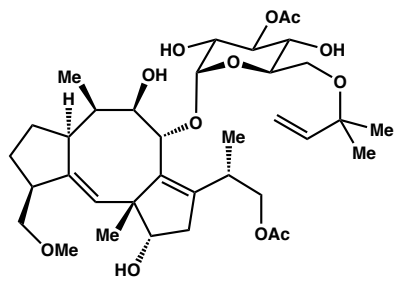

fusicoccin $A(F C)$
C-terminal 14-3-3 recognition motif

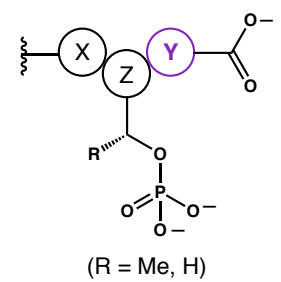

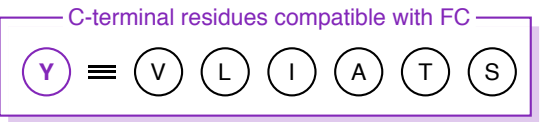

Figure 1. Fusicoccin A (FC) is a toxin produced by fungus Fusicoccum amygdali. This cell-permeable diterpene glycoside is a stabilizer of 14-3-3 PPIs that utilize a C-terminal recognition motif $(\mathrm{X}=$ any residue; $\mathrm{pZ}=$ phosphorylated $\mathrm{S}$ or $\mathrm{T} ; \mathrm{Y}=\mathrm{V}, \mathrm{L}, \mathrm{I}$, $\mathrm{A}, \mathrm{T}$, or $\mathrm{S}$ ) for molecular recognition.

multiple studies linking FC-induced stabilization of 14-3$3 \cdot$ client phosphoprotein contacts in vitro to modified client function in cell culture. ${ }^{16,17-23}$ These investigations have established that FC is selective for 14-3-3 PPIs that rely on the atypical C-terminal phosphorylation recognition motif $\mathrm{XpZY}_{\mathrm{COOH}}$ for molecular recognition, and also critically, that FC cannot effectively bind 14-3-3 PPIs involving the more common RXXpZXP consensus sequence. ${ }^{22,24}$ The identity of the C-terminal residue (Y) within this alternative motif is critical for FC activity. ${ }^{25}$ Recently, we demonstrated that small branched residues are required at this position, and leveraging this observation, we developed a comprehensive list of candidate 14-3-3 PPI targets for FC. ${ }^{26}$

Together, the aforementioned studies provide a clear picture of the requirements for interactions between $\mathrm{FC}$ and client proteins within the 14-3-3 binding groove. However, the effect of different 14-3-3 isoforms on FC activity remains an important open-ended question. There are seven isoforms of 14-3-3 in humans $(\alpha, \beta, \varepsilon, \zeta, \tau, \eta$ and $\gamma)$, each expressed by a distinct gene. Detailed structural comparisons show that the amino acid sequences and overall structures of 14-3-3 
Table 1. Distribution and isoform-specific functions of human 14-3-3 isoforms.

\begin{tabular}{|c|c|c|c|}
\hline isoform (gene) & tissue localization & isoform-specific roles in disease & reference \\
\hline$\sigma(\mathrm{SFN})$ & $\begin{array}{l}\text { lung, breast, uterus, ovary, blood, } \\
\text { skin, liver, pancreas, cornea }\end{array}$ & $\begin{array}{l}\text { - Epigenetically suppressed in epithelial carcinomas } \\
\text { - Functions as tumor suppressor }\end{array}$ & $9,28,31$ \\
\hline$\beta(Y W H A B)$ & $\begin{array}{l}\text { brain, lung, colon, gastric lining, } \\
\text { liver, bladder, kidney }\end{array}$ & - Overexpressed in squamous cell carcinoma & 28,35 \\
\hline$\varepsilon(\mathrm{YWHAE})$ & $\begin{array}{l}\text { brain (hippocampus), renal, liver, } \\
\text { breast, gastric lining }\end{array}$ & $\begin{array}{l}\text { - YWHAE deleted in Miller-Dieker syndrome } \\
\text { - Found in Lewy Bodies from Parkinson's patients }\end{array}$ & 10,36 \\
\hline$\zeta($ YWHAZ) & $\begin{array}{l}\text { brain, breast, lung, colon, head and } \\
\text { neck, oral, ovary, esophagus }\end{array}$ & $\begin{array}{l}\text { - Overexpressed in cancer and correlates with poor prognosis } \\
\text { - Found in NTF's from Alzheimer's patents; binds to Tau }\end{array}$ & $28,32,33$ \\
\hline$\tau(Y W A H Q)$ & $\begin{array}{l}\text { brain (frontal cortex), breast, lung, } \\
\text { prostate }\end{array}$ & $\begin{array}{l}\text { - Overexpression protects from dopaminergic cell loss } \\
\text { - Diminished expression in Alzheimer's patients }\end{array}$ & 10,34 \\
\hline$\eta($ YWHAH) & $\begin{array}{l}\text { brain (frontal cortex), liver, lung, } \\
\text { prostate }\end{array}$ & $\begin{array}{l}\text { - Binding to } \alpha \text {-synuclein disrupted in Parkinson's disease } \\
\text { - Diminished expression in Alzheimer's patients }\end{array}$ & 10,34 \\
\hline$\gamma(\mathrm{YWHAG})$ & brain, breast, liver, lung & $\begin{array}{l}\text { - Correlated with Amyotrophic lateral sclerosis (ALS) } \\
\text { - Overexpressed in lung cancer; p53 reduces14-3-3 } \gamma \text { mRNA }\end{array}$ & $10,28,37$ \\
\hline
\end{tabular}

isoforms are highly conserved, especially within in the phospho-binding amphipathic groove. ${ }^{3,6}$ This similarity is reflected in the ability of one isoform to compensate for another in certain settings and suggests functional overlap between 14-3-3 isoforms. ${ }^{27}$ Nevertheless, as summarized in Table 1, there is emerging evidence for both tissue-specific expression of 14-3-3 isoforms and isoform-specific functions. ${ }^{28-30}$ The most distinct isoform is $14-3-3 \sigma$, which is expressed in epithelial cells and acts as a tumor suppressor by promoting DNA repair pathways. ${ }^{9,31}$ In contrast, the other 143-3 isoforms promote cell survival. For example, overexpression of $14-3-3 \xi$ is common in many cancers and depletion of this isoform slows tumor growth in rodent models following treatment with cisplatin. ${ }^{32,33}$ Similarly, $14-3-3 \tau$ and $14-3-3 \eta$ are the predominant isoforms found in neurons within the frontal cortex and their expression levels are significantly reduced in brain samples taken from Alzheimer's patients. ${ }^{34}$ The underlying cause for the differential expression patterns of 14-3-3 isoforms remains to be determined, and similarly, the extent to which isoform-dependent interactions with FC contribute to the pharmacology of this chemotype is unknown. Herein, we report the isoform-specificity profile of $\mathrm{FC}$ in vitro using recombinant human 14-3-3 isoforms and a focused set of fluorescein-labeled hexaphosphopeptides mimicking the Cterminal 14-3-3 recognition domains of client proteins targeted by $\mathrm{FC}$ in human cell culture.

Our studies commenced with an analysis of the available structural data for FC in complex with human $14-3-3 \cdot$ client protein complexes. We used the crystal structure (PDB 4DJJ) of human 14-3-3 $\sigma$ in complex with FC and a C-terminal phosphorylated peptide from human estrogen receptor alpha $(E R \alpha)$ as a model to map 14-3-3 residues within the natural product and peptide-binding groove (Figure 2). ${ }^{21}$ The overall structures of 14-3-3 isoforms have been reviewed, and thus, only a summary of pertinent features related to $\mathrm{FC}$ interactions are given here. ${ }^{5,6}$ The monomeric unit of 14-3-3 $\sigma$ is comprised of a bundle of nine $\alpha$-helices ( $\alpha \mathrm{A}$ to $\alpha \mathrm{I}$ ). As shown in Figure 2A, FC and the ER $\alpha$ phospholigand (sequence: GFPApTV $\mathrm{COOH}_{\mathrm{COH}}$ ) locate to the amphiphilic 14-3-3 binding groove (green), which largely is comprised of the $\alpha$-helices $\alpha \mathrm{C}, \alpha \mathrm{E}, \alpha \mathrm{G}$ and $\alpha \mathrm{I}$. The ERa phosphopeptide partially fills this groove and is held in place via contacts formed between the pendant phosphothreonine and a triad of polar residues $(\mathrm{R}$ -
56, R-129, and Y-130) within the 14-3-3 binding groove that form a phospho-binding pocket (Figure 2B). Moving up the binding groove, hydrogen bonds (2.5-2.9 $\AA$ ) formed between the phospholigand and both K-49 and N-175 on 14-3-3 are also apparent. Notably, one would expect these interactions to be conserved for any C-terminal phospholigand docking to 14-

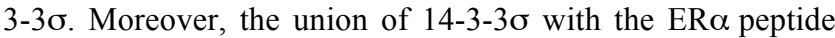
creates a hydrophobic pocket at the interface of the PPI where FC can bind. Binding of the natural product is driven largely by hydrophobic contacts with both protein partners. ${ }^{11}$ As previously discussed, the C-terminal residue of ER $\alpha$ makes a key hydrophobic contact with the puckered 5-8-5 carbotricycle of FC. ${ }^{26}$ In contrast, contacts with $14-3-3$ are limited to the convex periphery of the terpene subunit and include three hydrogen bonds with 14-3-3 residues: (1) K-122 on 14-3-3 and the A-ring methylmethoxy group on FC; (2) D-215 of 143-3 and the $\mathrm{C}_{8}$ alcohol of $\mathrm{FC}$; and (C) a water-mediated hydrogen bond formed between N-42 of 14-3-3 and the sugar moiety of FC. Importantly, a sequence comparison of the different human 14-3-3 isoforms demonstrates a remarkably high degree of residue conservation within the phospholigand binding groove (Figure 2B). This similarity in 14-3-3 contrasts with the diversity of $\mathrm{C}$-terminal client protein sequences that are compatible with FC. ${ }^{26}$ Nevertheless, our sequence and structural analysis unambiguously show that residues of 14-33 contacting both FC (including D-122, D215 and N-42) and $\mathrm{ER} \alpha$ (including R-56, R-129, Y-130, K-49 and N-175) are strictly conserved across all human isoforms.

To understand the extent to which the observed conservation of residues within the 14-3-3 phospholigand binding groove leads to similarities in the strength of PPI stabilization provided by $\mathrm{FC}$, we determined $\mathrm{EC}_{50}$ values for FC across a subset of human 14-3-3 isoforms using a suite of three client phosphoproteins, namely, ER $\alpha,{ }^{21}$ Task $3^{22}$ and Gplbo. $^{23}$ These clients were selected because they are wellestablished targets for stabilization by FC, and because their sequences are variable with regard to relative hydrophobicity, number of charged residues, identity of the phosphorylated side chain ( $\mathrm{S}$ or $\mathrm{T}$ ), and identity of the $\mathrm{C}$-terminal residue.

As shown in Figure 3, fluorescence polarization assays using fluorescein-labeled, C-terminal hexaphosphopeptide (ctp) motifs replicating the functional 14-3-3 binding domains of human ER $\alpha$ (1), Task3 (2) and Gplbo (3) provided 


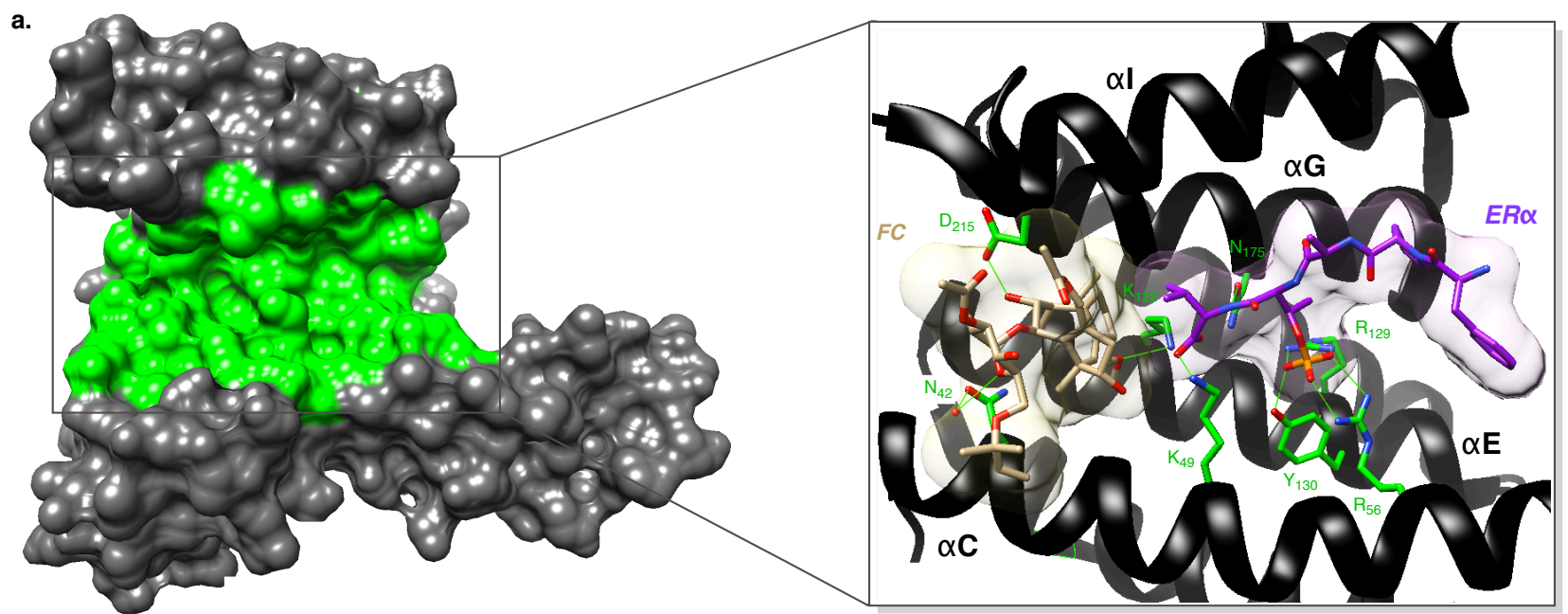

hydrogen bonds formed between 14-3-3 $\sigma$ and small molecules within the phospholigand binding groove

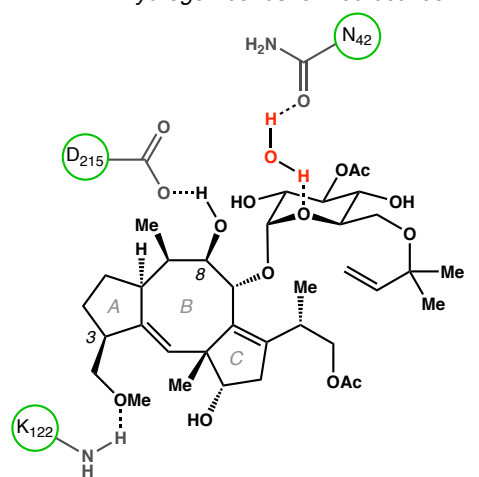

14-3-3б:FC contacts

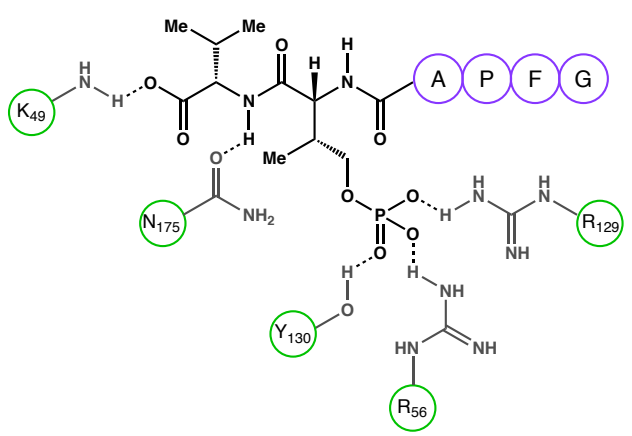

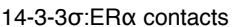

b. sequence homology within the phospholigand binding groove

$$
\alpha C: E_{39}-R_{60} \quad \alpha E: E_{115}-E_{133} \quad \alpha G: P_{167}-I_{183} \quad \alpha I: N_{214}-L_{229}
$$

14-3-30 EERNLLSVAYKNNVGGQRAAWR ESRVFYKKMKGDYYRYLAE PIRLGLALN

14-3-3 EERNLLSVAYKNVVGARRSSWR ESKVFYLKMKGDYFRYLSE PIRLGLALNFSVFYYEI KDSTLIMQLLRDNLTL

14-3-3 $\varepsilon$ EERNLLSVAYKNVIGARRASWR ESKVFYYKMKGDYHRYLAE PIRLGLALNFSVFYYEI KDSTLIMQLLRDNLTL

14-3-3ל EERNLLSVAYKNVVGARRSSWR ESKVFYLKMKGDYYRYLAE PIRLGLALNFSVFYYEI KDSTLIMQLLRDNLTL

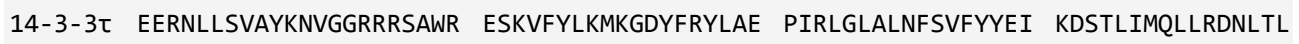

14-3-3n EDRNLLSVAYKNVVGARRSSWR ESKVFYLKMKGDYYRYLAE PIRLGLALNFSVFYYEI KDSTLIMQLLRDNLTL

14-3-3 $\gamma$ EERNLLSVAYKNVVGARRSSWR ESKVFYLKMKGDYYRYLAE PIRLGLALNYSVFYYEI KDSTLIMQLLRDNLTL

Figure 2. Structural and sequence analysis of the $14-3-3 \sigma \cdot E R \alpha \bullet F C$ ternary complex (PDB 4JDD). (a) Renderings of the ternary complex showing the 14-3-3 phospholigand binding groove (green) and key interactions between 14-3-3 $\sigma$ and both FC and ER $\alpha$. (b) Sequence homology analysis of the phospholigand-binding groove across all human isoforms revealing a high degree of residue conservation (fully conserved residues shown in blue) in this region.

complete dose-response curves that allowed us to extract $\mathrm{EC}_{50}$ values for FC-induced stabilization of each peptide in association with five recombinant human 14-3-3 isoforms. With both ER $\alpha$-ctp and Task3-ctp as clients, the $\mathrm{EC}_{50}$ values for FC were similar to one another across all isoforms, demonstrating a lack of specificity for FC association with the binary complex between individual 14-3-3 isoforms and these two peptides. In contrast, using the Gplb $\alpha$-ctp, we observed notable isoform-specific differences in $\mathrm{EC}_{50}$ values for $\mathrm{FC}$. The lowest $\mathrm{EC}_{50}$ value, $5.2 \pm 1.4 \mu \mathrm{M}$, was obtained with 14-3$3 \xi$, whereas the highest $\mathrm{EC}_{50}$ values, $25 \pm 1.4 \mu \mathrm{M}$ and $25 \pm 3.6$ $\mu \mathrm{M}$, were observed with $14-3-3 \varepsilon$ and $14-3-3 \sigma$, respectively. While modest in magnitude, this approximately 5-fold difference lies outside the standard deviation of our measurements and demonstrates that, for certain client phosphoproteins, FC exhibits a 14-3-3 isoform preference.
Next, we investigated the extent to which the intrinsic affinity of individual client phosphopeptides varies across 143-3 isoforms using an established fluorescence polarization assay. $^{22,26}$ Thus, $100 \mathrm{nM}$ solutions of phospholigands $\mathbf{1}-\mathbf{3}$ were titrated with individual 14-3-3 isoforms to establish the affinity (apparent $\mathrm{K}_{\mathrm{d}}$ ) of each PPI in the absence of FC. The results of these experiments revealed unexpected differences in 14-3-3 isoform preferences for individual client phospholigands (Table 2). Titrations using the ER $\alpha$-ctp (1) showed that 14-3-3 $\beta$ had the strongest affinity (apparent $K_{d}$ $=0.8 \pm 0.1 \mu \mathrm{M})$, whereas the interaction using 14-3-3 $\sigma$ was comparatively weaker (apparent $\mathrm{K}_{\mathrm{d}}=6.1 \pm 0.6 \mu \mathrm{M}$ ). This represents a surprising 7.6-fold preference between the two isoforms. In contrast, for Task3-ctp (2), 14-3-3 $\zeta$ showed the 

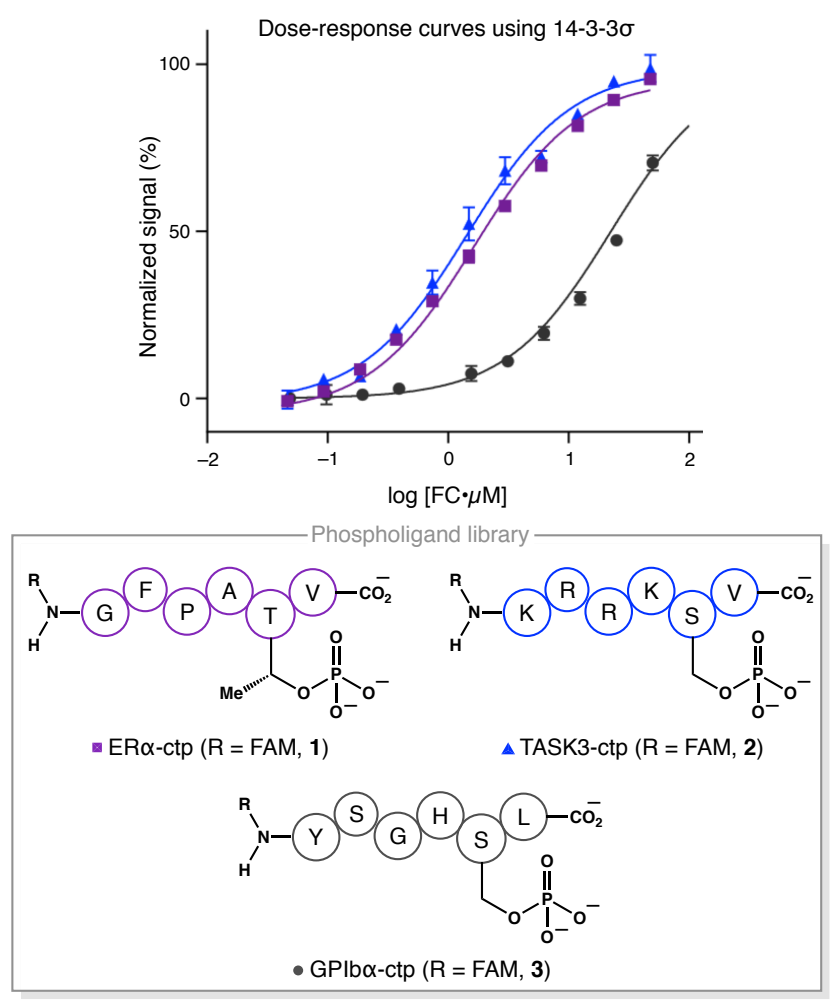

$\begin{array}{cccc}\begin{array}{c}\text { 14-3-3 } \\ \text { isoform }\end{array} & \text { ER } \alpha \text {-ctp (1) } & \text { Task3-ctp (2) } & \text { Gplbo-ctp (3) } \\ \beta & 1.3 \pm 1.1 & 2.2 \pm 1.1 & 13 \pm 1.4 \\ \varepsilon & 2.3 \pm 1.1 & 1.1 \pm 1.1 & 25 \pm 1.4 \\ \zeta & 2.4 \pm 1.1 & 1.9 \pm 1.1 & 5.2 \pm 1.4 \\ \sigma & 3.5 \pm 1.0 & 3.0 \pm 1.1 & 25 \pm 3.6 \\ \tau & 1.8 \pm 1.1 & 1.8 \pm 1.1 & 21 \pm 2.0\end{array}$

Figure 3. Summary of dose-response experiments for FC. Dose-

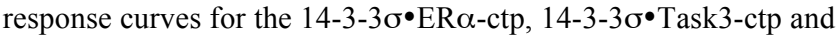
14-3-3 $\sigma^{\bullet}$ GPIb $\alpha$-ctp PPIs are shown. Structures and sequences of client FAM-labeled hexaphosphopeptides (ctp) replicating the 143-3 recognition domains of ER $\alpha$ (1), Task3 (2) and GPIb $\alpha$ (3). Complied $\mathrm{EC}_{50}(\mu \mathrm{M})$ extracted from the dose-response curves were generated from phospholigands 1-3 across five human 14-33 isoforms are provided (see the SI for details).

highest affinity (apparent $\mathrm{K}_{\mathrm{d}}=1.4 \pm 0.1 \mu \mathrm{M}$ ), whereas 14-3-3e showed the lowest affinity (apparent $\mathrm{K}_{\mathrm{d}}=6.2 \pm 0.5 \mu \mathrm{M}$ ). Although this 4.4-fold difference in isoform preference is less than we observed using the ER $\alpha$-ctp, it is nonetheless significant in light of the standard deviation of our measurements. Finally, we found that the results using Gplboctp mirrored the trends observed with Task3-ctp. Specifically, 14-3-3 $\zeta$ exhibited the highest affinity (apparent $\mathrm{K}_{\mathrm{d}}=16 \pm 3.0$ $\mu \mathrm{M}$ ), whereas $14-3-3 \varepsilon$ showed the lowest affinity (apparent $\mathrm{K}_{\mathrm{d}}$ $=63 \pm 12.0 \mu \mathrm{M})$. It should be noted, however, that the standard deviation of data collected for Gplba-ctp is significantly larger than with other phospholigands, presumably because this peptide binds to 14-3-3 weakly relative to ER $\alpha$-ctp and Task3-ctp. Together, these data demonstrate small, yet potentially relevant, preferences of individual client proteins for specific human 14-3-3 isoforms.

To examine whether the differential intrinsic affinities noted above were also manifested in isoform specific FC-
Table 2. ${ }^{a}$ Intrinsic affinity (apparent $\mathrm{K}_{\mathrm{d}}$ ) of phospholigands $\mathbf{1}$ 3 across 14-3-3 isoforms

$\begin{array}{cccc}\begin{array}{c}\text { 14-3-3 } \\ \text { isoform }\end{array} & \text { ER } \alpha \text {-ctp (1) } & \text { Task3-ctp (2) } & \text { Gplb } \alpha \text {-ctp (3) } \\ \beta & 0.8 \pm 0.07 & 2.5 \pm 0.14 & 22 \pm 7.5 \\ \varepsilon & 3.9 \pm 0.15 & 6.2 \pm 0.50 & 63 \pm 12 \\ \zeta & 1.5 \pm 0.27 & 1.4 \pm 0.10 & 16 \pm 3.0 \\ \sigma & 6.1 \pm 0.56 & 2.2 \pm 0.13 & 23 \pm 9.7 \\ \tau & 1.3 \pm 0.10 & 2.2 \pm 0.27 & 30 \pm 2.9\end{array}$

${ }^{a}$ Reported $K_{d}(\mu M)$ values represent the average of two independent experiments.

induced stabilization of 14-3-3 PPIs, we repeated our titrations of client phospholigands 1-3 with 14-3-3 isoforms in the presence of $80 \mathrm{mM}$ FC. As expected based on our previous study, we found that FC stabilizes all three client phosphopeptides across the five human 14-3-3 isoforms investigated by at least one order of magnitude (Figure 4 ). ${ }^{26}$ In addition, we also observed differences in isoform preference trends relative to the titrations carried out in the absence of FC. Thus, for ER $\alpha$-ctp, 14-3-3 $\beta$ showed the highest FCstabilized affinity (apparent $\mathrm{K}_{\mathrm{d}}=0.04 \pm 0.01 \mu \mathrm{M}$ ), whereas 14-3-3 $\tau$ was the weakest binder (apparent $K_{d}=0.13 \pm 0.01$ $\mu \mathrm{M})$. In this case, the difference between the best and worst performing isoform (3.3-fold) is notably less than observed for the same titrations carried out in the absence of FC (7.6-fold). In contrast, for Task3-ctp, 14-3-3 $\xi$ and 14-3-3 $\sigma$ exhibited the highest affinity (apparent $K_{d}=0.06 \pm 0.01 \mu \mathrm{M}$ for both isoforms), whereas $14-3-3 \varepsilon$ showed the lowest affinity (apparent $\mathrm{K}_{\mathrm{d}}=0.14 \pm 0.03 \mu \mathrm{M}$ ). Again, similar to ER $\alpha$-ctp, the magnitude of the isoform-specificity was minimized (2.2fold) in presence of FC. The same trend was observed for the Gplbo-ctp; 14-3-3 $\zeta$ showed the highest affinity (apparent $\mathrm{K}_{\mathrm{d}}=$ $0.50 \pm 0.25 \mu \mathrm{M}$ ) and 14-3-3 $\sigma$ had the lowest affinity (apparent $\left.\mathrm{K}_{\mathrm{d}}=1.7 \pm 0.30 \mu \mathrm{M}\right)$, resulting in 3.4 -fold preference between these isoforms

When combined with the intrinsic affinities of client phosphopeptides measured in the absence of FC (Table 2), the data presented in Figure 4 also provide a measure of the extent to which FC stabilizes the interaction between each phospholigand/14-3-3 isoform combination. The resultant fold-stabilization $\left(\mathrm{S}_{\mathrm{F}}\right)$ for each PPI is summarized by the histogram shown in Figure 4. Analysis of these data reveals several noteworthy trends. Considering initially the stabilization of individual client phosphoproteins across 14-33 isoforms, we determined that for the ER $\alpha$-ctp, FC provides 5.4-fold greater stabilization with 14-3-3 $\sigma$ compared to the

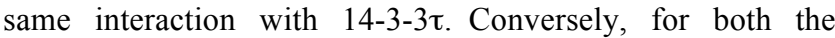
Task3-ctp and Gplbo-ctp phosphopeptides, FC exhibits less than 3-fold differential stability between the highest and lowest affinity isoforms. In addition, the isoform with the lowest intrinsic affinity for each client phosphopeptide in Table 2 exhibits that highest degree of stabilization in the presence of FC (Figure 4, bold). In these cases, the activity of $\mathrm{FC}$ is impressive. For example, FC induces a 39 -fold increase in affinity for the $14-3-3 \varepsilon^{\bullet} \mathrm{Gplb} \alpha$-ctp PPI, from an intrinsic apparent $\mathrm{K}_{\mathrm{d}}$ of $63 \pm 12 \mu \mathrm{M}$ to a stabilized apparent $\mathrm{K}_{\mathrm{d}}$ of $1.6 \pm$ $0.18 \mu \mathrm{M}$. Finally, analysis of differential FC-induced stabilization across $14-3-3$ isoforms suggests that $14-3-3 \sigma$ is distinct. For example, FC provides 4.2-fold greater 


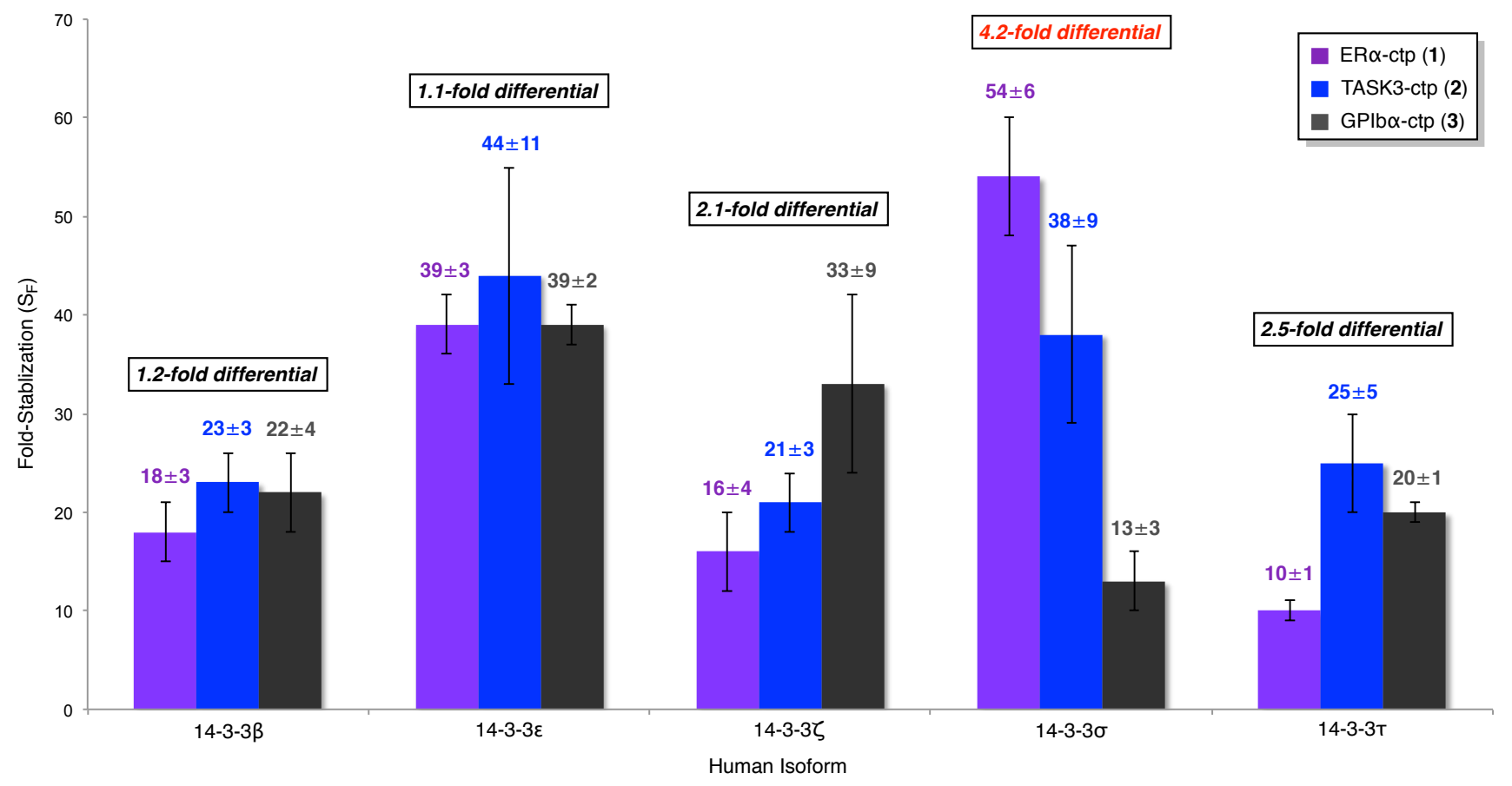

$\begin{array}{ccccccc}\text { 14-3-3 isoform } & \text { ER } \alpha \text {-ctp (1) } & \mathrm{S}_{\mathrm{F}} & \text { Task3-ctp (2) } & \mathrm{S}_{\mathrm{F}} & \text { Gplbo-ctp (3) } & \mathrm{S}_{\mathrm{F}} \\ \beta & 0.04 \pm 0.01 & 18 \pm 3 & 0.11 \pm 0.01 & 23 \pm 3 & 1.0 \pm 0.26 & 22 \pm 4 \\ \varepsilon & 0.10 \pm 0.01 & 39 \pm 3 & 0.14 \pm 0.03 & \mathbf{4 4} \pm \mathbf{1 1} & 1.6 \pm 0.18 & \mathbf{3 9} \pm \mathbf{2} \\ \zeta & 0.09 \pm 0.02 & 16 \pm 4 & 0.06 \pm 0.01 & 21 \pm 3 & 0.50 \pm 0.25 & 33 \pm 9 \\ \sigma & 0.11 \pm 0.01 & \mathbf{5 4} \pm \mathbf{6} & 0.06 \pm 0.01 & 38 \pm 9 & 1.7 \pm 0.30 & 13 \pm 3 \\ \tau & 0.13 \pm 0.01 & 10 \pm 1 & 0.09 \pm 0.01 & 25 \pm 5 & 1.5 \pm 0.10 & 20 \pm 1\end{array}$

${ }^{\mathrm{a}}$ Reported $\mathrm{K}_{\mathrm{d}}(\mu \mathrm{M})$ values represent the average of two independent experiments carried out in the presence of $80 \mu \mathrm{M}$ FC. ${ }^{\mathrm{b}}$ Fold-stabilization $\left(\mathrm{S}_{\mathrm{F}}\right)$ was determined by diving the intrinsic apparent $\mathrm{K}_{\mathrm{d}}$ reported in Table 2 by the stabilized apparent $\mathrm{K}_{\mathrm{d}}$ shown above.

Figure 4. ${ }^{a, b}$ Affinity (apparent $\mathrm{K}_{\mathrm{d}}$ ) and fold-stabilization $\left(\mathrm{S}_{\mathrm{F}}\right)$ of phospholigands 1-3 across 14-3-3 isoforms in the presence of FC.

stabilization for $14-3-3 \mathrm{~s} \bullet$ ER $\alpha$-ctp PPI $(54 \pm 6)$ than the $14-3$ $3 \mathrm{~s} \bullet \mathrm{Gplb} \alpha$-ctp PPI $(13 \pm 3)$. Although the overall magnitude of this differential stability is modest, it sharply contrasts with phospholigands $\mathbf{1}-\mathbf{3}$ across the remaining isoforms, where the differential of FC-induced stabilization was markedly less variable ( $\leq 2.5$-fold differential). Together, these data demonstrate that FC stabilizes interactions between ER $\alpha$-ctp, Task3-ctp, and Gplb $\alpha$-ctp to a similar extent across all 14-3-3 isoforms, with the notable exception of 14-3-3 $\sigma$, which appears to be an outlier in our study.

In conclusion, we have established the 14-3-3 isoformspecificity profile of PPIs stabilized by fusicoccin A (FC), a natural product that has recently amassed attention as a chemical probe of 14-3-3 functions. Our sequence analysis of human 14-3-3 isoforms, along with inspection of available crystallographic data, demonstrate a remarkable degree of conservation of residues that contact both FC and client phospholigands within the 14-3-3 binding groove. Based on these observations, one would expect both the intrinsic affinities of client proteins and FC-induced stabilization of 143-3 client protein complexes to be very similar across the 143-3 isoforms. In contrast, our results reveal a modest, yet unexpected, level of isoform preferences for individual client phospholigands. These isoform-specific effects were most notable in the absence of FC; however, our data suggests that FC differentially stabilizes PPIs involving 14-3-3 $\sigma$. These isoform-dependent activities might be enhanced via rational design of synthetic, non-natural FC analogs. We believe there is strong motivation for such work, especially in light of the fact that 14-3-3o possesses isoform-specific functions as a tumor suppressor in human cell culture. . $^{1,39}$

\section{ASSOCIATED CONTENT}

\section{Supporting Information}

The Supporting Information is available free of charge on the ACS Publications website.

Materials and methods, supplementary fluorescence-anisotropy data and multiple sequence alignment for 14-3-3 isoforms.

\section{AUTHOR INFORMATION}

\section{Corresponding Author}

James H. Frederich - Department of Chemistry and Biochemistry, Florida State University, Tallahassee, Florida 32306, United States

Email: frederich@chem.fsu.edu.

\section{Authors}

Ananya Sengupta - Department of Chemistry and Biochemistry, Florida State University, Tallahassee, Florida 32306, United States 
Josue Liriano - Department of Chemistry and Biochemistry, Florida State University, Tallahassee, Florida 32306, United States

Ewa A. Bienkiewicz - Department of Biomedical Sciences, College of Medicine, Florida State University, Tallahassee, Florida 32306, United States

Brian G. Miller - Department of Chemistry and Biochemistry, Florida State University, Tallahassee, Florida 32306, United States

\section{ACKNOWLEDGMENT}

This investigation was funded by the National Institutes of Health (R01-GM125926 to JHF and R01-GM133843 and R01GM115388 to BGM) and the Pfeiffer Endowed Professorship for Cancer Research (BGM). We acknowledge B. Stefanovic (FSU) for access to his microplate reader.

\section{REFERENCES}

(1) Jones, S.; Thornton, J. M. Principals of protein-protein interactions. Proc. Natl. Acad. Sci. USA 1996, 93, 13-20.

(2) Seet, B. T.; Dikic, I.; Zhou, M. M.; Pawson, T. Reading protein modifications with interaction domains. Nat. Rev. Mol. Cell Bio. 2006, 7, 473-483.

(3) Pennington, K. L.; Chan, T. Y.; Torres, M. P.; Andersen, J. L. The dynamic and stress-adaptive signaling hub of 14-3-3: emerging mechanisms of regulation and context-dependent protein-protein interactions. Oncogene 2018, 37, 5587-5604.

(4) Johnson, C.; Crowther, S.; Stafford, M. J.; Campbell, D. G.; Toth, R.; MacKintosh, C. Bioinformatic and experimental survey of 14-3-3 binding sites. Biochem. J. 2010, 427, 69-78.

(5) Yang, X.; Lee, W. H; Sobott, F.; Papgrigoriou, E.; Robinson, C. V.; Grossmann, J. G.; Sundström, M.; Doyle, D. A.; Elkins, J. M. Structural basis for protein-protein interactions in the 14-3-3 protein family. Proc. Natl. Acad. Sci. USA 2006, 103, 17237-17242.

(6) Gardino, A. K.; Smerdon, S. J.; Yaffe, M. B. Structural determinants of 14-3-3 binding specificities and regulation of subcellular localization of 14-3-3-ligand complexes: a comparison of the X-ray structures of all human 14-3-3 isoforms. Semin. Cancer Biol. 2006, 16, 173-182.

(7) Yaffe M, B. How do 14-3-3 proteins work? Gatekeeper phosphorylation and the molecular anvil hypothesis. FEBS Lett. 2002, 513, 53-57.

(8) Ottmann, C.; Marco, S.; Jaspert, N.; Marcon, C.; Schauer, N.; Weyand, M.; Vandermeeren, C.; Duby, G.; Boutry, M.1 Wittinghofer, A.; Rigaud, J.; Oecking, C. Structure of a 14-3-3 coordinated hexamer of the plant plasma membrane $\mathrm{H}^{+}$-ATPase by combining X-ray crystallography and electron cryomicroscopy. Mol. Cell. 2007, 25, 427-440.

(9) Hermeking, H. The 14-3-3 cancer connection. Nat. Rev. Cancer. 2003, 3, 931-943.

(10) Foote, M.; Zhou, Y. 14-3-3 proteins in neurological disorders. Int. J. Biochem. Mol. Bio. 2012, 3, 152-164.

(11) Stevens, L. M.; Sijbesma, E.; Botta, M.; MacKintosh, C.; Obsil, T.; Landrieu, I.; Cau, Y.; Wilson, A. J.; Karawajczyk, A.; Eickhoff, J.; Davis, J.; Hann, M.; O’Mahony, G.; Doveston, R. G.; Brunsveld, L.; Ottmann, C.. Modulators of 14-3-3 protein-protein interactions. $J$. Med. Chem. 2018, 61, 3755-3778.

(12) Turner, N. C.; Graniti, A. Fusicoccin: a fungal toxin that opens stomata. Nature 1969, 223, 1070-1071.

(13) Fullone, M. R.; Visconti, S.; Marra, M.; Fogliano, V.; Aducci, P. Fusicoccin effect on the in vitro interaction between plant 14-3-3 and plasma membrane $\mathrm{H}^{+}$ATPase. J. Biol. Chem. 1998, 273, 7698-7702.

(14) Wurtele, M.; Jelich-Ottmann, C; Wittinghofer, A.; Oecking, C. Structural view of a fungal phytotoxin acting on a 14-3-3 regulatory complex. EMBO J. 2003, 22, 987-994.

(15) deVries-van Leeuwen, I. J.; Kortekaas-Thijssen, C.; Mandouckou J. A. N.; Kas, S.; Evidente, A.; de Boer, A. H. Fusicoccin A selectively induces apoptosis in tumor cells after interferon-alpha priming. Cancer Lett. 2010, 293, 198-206.
(16) Kaplan, A.; Morquette, B.; Kroner, A.; Leong, S.; Madwar, C.; Sanz, R.; Banerjee, S. L.; Antel, J.; Bisson, N.; David, S.; Fournier, A. E. Small-molecule stabilization of 14-3-3 protein-protein interactions stimulates axon regeneration. Neuron 2017, 93, 1082-1093.

(17) Andrei, S. A.; de Vink, P.; Sijbesma, E.; Han, L.; Brunsveld, L.; Kato, N.; Ottmann, C.; Higuchi, Y. Rationally designed semisynthetic natural product analogs for stabilization of 14-3-3 protein-protein interactions. Angew. Chem., Int. Ed. 2018, 57, 13470-13474.

(18) Stevers, L. M.; Lam, C. V.; Leysen, S. F.; Meijer, F. A.; van Scheppingen, D. S.; de Vries, R. M.; Carlile, G. W.; Milroy, L. G.; Thomas, D. Y.; Brunsveld, L.; Ottmann, C. Characterization and small-molecule stabilization of the multisite tandem binding between 14-3-3 and the R domain of CFTR. Proc. Natl. Acad. Sci. USA 2016, 113, E1152-1161.

(19) Doveston, R. G.; Kuusk, A.; Andrei, S. A.; Leysen, S.; Cao, Q.; Castaldi, M. P.; Hendricks, A.; Brunsveld, L.; Chen, H.; Boyd, H.; Ottmann, C. Small-molecule stabilization of the p53-14-3-3 proteinprotein interaction. FEBS Lett. 2017, 591, 2449-2457.

(20) Scwarczynska, M.; Molzan, M.; Ottmann, C. Activation of NF$\mathrm{kB}$ signaling by fusicoccin-induced dimerization. Proc. Natl. Acad. Sci. USA. 2013, 110, E377-386.

(21) de Vries-van Leeuwen, I. J.; da Costa Pereira, D.; Flach, K. D.; Piersma, S. R.; Haase, C.; Bier, D.; Yalcin, X.; Michalides, R.; Feenstra, K. A.; Jimenez, C, R.; de Greef, T. F. A.; Brunsveld, L.; Ottmann, C.; Zwart, W.; de Boer, A. H. Interaction of 14-3-3 proteins with the estrogen receptor alpha $\mathrm{F}$ domain provides a drug target interface. Proc. Natl. Acad. Sci. USA 2013, 110, 8894-8899.

(22) Anders, C.; Higuchi, Y.; Koschinsky, K.; Bartel, M.; Schumacher, B.; Thiel, P.; Nitta, H.; Preisig-Muller, R.; Schlichthorl, G.; Renigunta, V.; Ohkanda, J.; Daut, J.; Kato, N.; Ottmann, C. A semisynthetic fusicoccane stabilizes a protein-protein interaction and enhances expression of $\mathrm{K}^{+}$channels at the cell surface. Chem. Biol. 2013, 20, 583-593.

(23) Camoni, L.; di Lucente, C.; Visconti, S.; Aducci, P. The phytotoxin fusicoccin promotes platelet aggregation via 14-3-3glycoprotein Ib-IX-V interaction. Biochem. J. 2011, 436, 429-436.

(24) Paiardini, A.; Aducci, P.; Cervoni, L.; Cutruzzola, F.; Di Lucente, C.; Janson, G.; Pascarella, S.; Rinaldo, S.; Visconti, S.; Camoni, L. The phytotoxin fusicoccin differentially regulates 14-3-3 proteins association to mode III targets. IUBMB Life 2014, 66, 52-62. (25) Ohkanda, J.; Kusumoto, A.; Punzalan, L.; Masuda, R.; Wang, C.; Parvatkar, P; Akase, D.; Aida, M.; Uesugi, M.; Higuchi Y.; Kato, N. Structural effects of fusicoccin upon upregulation of 14-3-3 phospholigand interaction and cytotoxic activity. Chem. Eur. J. 2018, 24, 16066-16071.

(26) Sengupta, A.; Liriano, J.; Miller, B. G.; Frederich, J. H; Analysis of interactions stabilized by fusicoccin A reveals an expanded suite of potential 14-3-3 binding partners. ACS Chem. Bio. 2020, 15, 305310 .

(27) Schoenwaelder, S. M.; Darbousset, R.; Cranmer, S. L.; Ramshaw, H. S.; Orive, S. L.; Sturgeon, S. Yan, Y.; Yao, Y.; Krycer, J. R.; Woodcock, J.; Maclean, J.; Pitson, S.; Zheng, X.; Henstridge, D. C.; van der Wal, D.; Gardiner, E. E.; Berndt, M. C.; Andrews, R. K.; James, D. E.; Lopez, A. F.; Jackson, S. P. 14-3-3ל regulates the mitochondrial respiratory reserve linked to platelet phosphatidylserine exposure and procoagulant function. Nat. Commun. 2016, 7, 12862 12866.

(28) Fan, X.; Cui, L.; Zeng, Y.; Song, W.; Gaur, U.; Yang, M. 14-3-3 proteins are on the crossroads of cancer, aging, and age-related neurodegenerative disease. Int. J. Mol. Sci. 2019, 20, 3518-3539.

(29) Qi, W.; Liu, X.; Qiao, D.; Martinez, J. D. Isoform-specific expression of 14-3-3 proteins in human lung cancer tissues. Int. J. Cancer 2005, 113, 359-363.

(30) Chandra, S.; Fornai, F.; Kwon, H. B.; Yazdani, U.; Atasoy, D.; Liu, X.; Hammer, R. E.; Battaglia, G.; German, D. C.; Castillo, P. E.; Sudhof, T. C. Double-knockout mice for alpha- and beta-synucleins: effect on synaptic functions. Proc. Natl. Acad. Sci. USA 2004, 101, 14966-14971. 
(31) Chan, T. A.; Hermeking, H.; Legauer, C.; Kinzler, K. W.; Vogelstein, B. 14-3-3 $\sigma$ is required to prevent mitotic catastrophe after DNA damage. Nature 1999, 401, 616-620.

(32) Neal, C. L.; Xu, J.; Li, P.; Mori, S.; Yang, J.; Neal, N. N.; Zhou, X.; Wyszomierski, S. L.; Yu, D. Overexpression of 14-3-3 $\zeta$ in cancer cells activates PI $3 \mathrm{~K}$ via binding the $\mathrm{p} 85$ regulatory subunit. Oncogene 2012, 31, 897-906.

(33) Yang, X.; Cao, W.; Zhou, J.; Zhang, W.; Zhang, X.; Lin, W.; Fei, Z.; Lin, H.; Wang, B. 14-3-3 $\zeta$ positive expression is associated with a poor prognosis in patients with glioblastoma. Neurosurgery 2011, 68, 932-938.

(34) Gu, Q.; Cuevas, E.; Raymick, J.; Kanungo, J.; Sarkar, S. Downregulation of 14-3-3 proteins in Alzheimer's Disease. Mol. Neurobiol. 2020, 57, 32-40.

(35) Wang, Z.; Nesland, J. M.; Sou, Z.; Trope, C. G.; Holm, R. The prognostic value of 14-3-3 isoforms in squamous cell carcinoma cases: $14-3-3 \beta$ and $\varepsilon$ are independent prognostic factors for these tumors. PLoS One 2011, 6, e24843.

(36) Sato, S; Chiba, T.; Sakata, E.; Kato, K.; Mizuno, Y.; Hattori, N.; Tanaka, K. $14-3-3 \eta$ is a novel regulator of parkin ubiquitin ligase. EMBO J. 2006, 25, 211-221.

(37) Spalice, A.; Parisi, P.; Nicita, F.; Pizzardi, G.; Del Balszo, F.; Iannetti, P. Neuronal migration disorders: clinical, neuroradiological $\backslash$ (38) Radhakrishnan V. M.; Putnam, C. W.; Qi, W.; Martinez, J. D. P53 suppresses expression of the 14-3-3 gamma oncogene. BMC Cancer. 2011, 11, 378-344.

(39) Li, Z.; Liu, J. Y.; Zhang, J. T. 14-3-3 Sigma, the double-edged sword of human cancers. Am. J. Trans. Res. 2009, 1, 326-340.

\section{TOC Graphic}

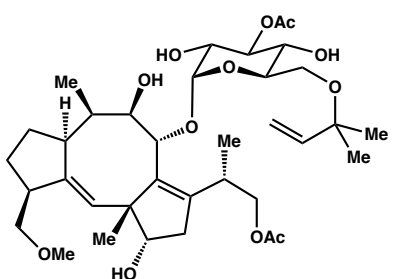

fusicoccin $\mathrm{A}(\mathrm{FC})$

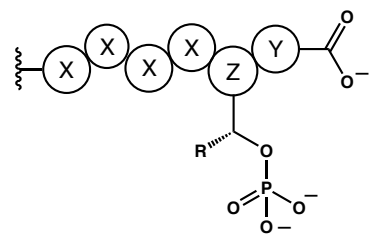

client phospholigand

$X=$ any residue, $Z=S / T, Y=V / L$

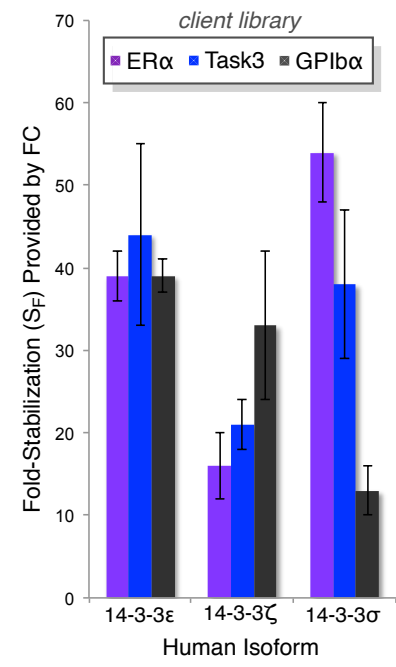

